\title{
The Influence of Atmosphere on Electrical Property of Copper Oxide Nanoparticles
}

\author{
Minghan Chen, Huichao Zhu, Xiaogan Li, Jun Yu, Haitao Cai, Xiaotong Quan, \\ Kaili Wang, and Jiaqi Zhang
}

School of Electronic Science and Technology, Faculty of Electronic Information and Electrical Engineering, Dalian University of Technology, Dalian 116024, China

Correspondence should be addressed to Huichao Zhu; zhuhuichao@dlut.edu.cn and Jun Yu; junyu@dlut.edu.cn

Received 29 October 2013; Accepted 8 January 2014; Published 18 February 2014

Academic Editor: Sheng-Joue Young

Copyright (C) 2014 Minghan Chen et al. This is an open access article distributed under the Creative Commons Attribution License, which permits unrestricted use, distribution, and reproduction in any medium, provided the original work is properly cited.

In the present work the authors have fabricated copper oxide nanoparticles through a water bath method and investigated their composition and microstructure using XRD and TEM methods. Their electrical properties were investigated under different atmospheres and temperatures after painting them onto the insulating substrates with interdigital $\mathrm{Pt}$ and Au electrodes. The electric currents are found dramatically dependent on the variation of humidity, temperature, and ratio of oxygen to nitrogen. In the end, the HCHO gas sensitivity of these nanoparticles has been investigated.

\section{Introduction}

Today, more and more attention has been paid to gas sensors based on semiconducting metal oxides for detection of hazardous, humid, flammable, or toxic gases, due to their rich resource, low cost, and easy fabrication. Plenty of ntype semiconducting metal oxides such as $\mathrm{ZnO}$ [1], $\mathrm{Fe}_{2} \mathrm{O}_{3}$ [2], $\mathrm{SnO}_{2}$ [3], $\mathrm{WO}_{3}[4]$, and $\mathrm{MoO}_{3}[5]$ are widely investigated due to their extensive sensing performance. Recently, increasing interest has been focused on p-type semiconducting metal oxides such as $\mathrm{CuO}$ [6] and $\mathrm{NiO}$ [7]. Copper oxides, including $\mathrm{CuO}$ and $\mathrm{Cu}_{2} \mathrm{O}$, are p-type semiconductors because of presence of acceptors levels attributed to copper vacancies. There have been some reports on $\mathrm{CuO}$ nanoparticles [8], nanowires [9], nanorods [10], nanoribbons [11], nanosheets [12], and microspheres [13] towards various target gases such as $\mathrm{NO}_{2}, \mathrm{H}_{2} \mathrm{~S}, \mathrm{C}_{2} \mathrm{H}_{5} \mathrm{OH}, \mathrm{CO}$, and $\mathrm{NH}_{3}$ in the working temperature ranging from $200^{\circ} \mathrm{C}$ to $400^{\circ} \mathrm{C}$. However, all synthesis methods of these copper oxide nanomaterials need harsh conditions such as high temperature or high pressure, no matter chemical vapor deposition, or hydrothermal process. There are few reports on copper oxide nanomaterials synthesized through low temperature conditions. In this study, we report the fabrication of copper oxide nanoparticles through a water bath process at relatively low temperature and normal pressure. We investigated influence of different atmospheres and temperatures on the electrical property of copper oxide nanoparticles.

\section{Experimental}

The copper oxide nanoparticles were synthesized using a water bath method under relatively low temperature and normal pressure [14]. The following chemical materials are employed in the synthesis process: copper (II) nitrate hemipentahydrate $\left(\mathrm{Cu}\left(\mathrm{NO}_{3}\right)_{2} \cdot 2.5 \mathrm{H}_{2} \mathrm{O}\right.$, analytical reagent), methenamine $\left(\left(\mathrm{CH}_{2}\right)_{6} \mathrm{~N}_{4}\right.$, analytical reagent), and deionized water. The synthesis was conducted by the thermal decomposition of $\mathrm{a} \mathrm{Cu}^{2+}$ amino complex with reagentgrade chemicals. Methenamine, $\left(\mathrm{CH}_{2}\right)_{6} \mathrm{~N}_{4}$, a nontoxic watersoluble and nonionic tetradentate cyclic tertiary amine was chosen to comply simultaneously with the precipitation of the divalent transition metal ion $\mathrm{Cu}^{2+}$ and the nucleation growth of its stable oxide form, copper (II) oxide. An equimolar $(0.1 \mathrm{M})$ aqueous solution (deionized water) of copper (II) nitrate hemipentahydrate and methenamine was prepared in a bottle with a screw cap. The bottle is then heated at a constant temperature of $100^{\circ} \mathrm{C}$ for 6 hours in a regular laboratory water bath kettle. Subsequently, the 


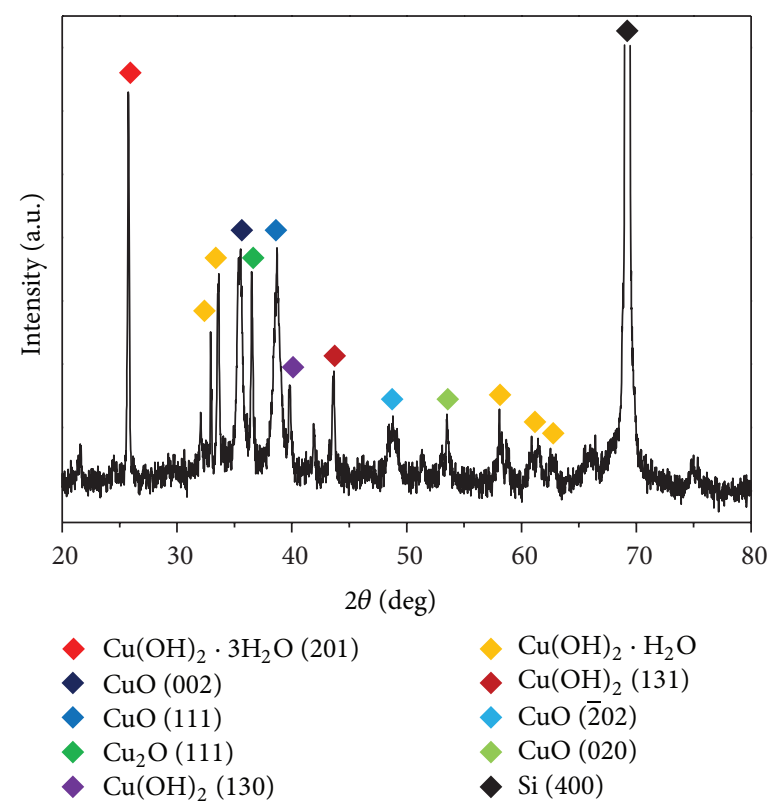

FIGURE 1: The XRD pattern of as grown copper oxide nanoparticles.

homogeneous nanoparticles are thoroughly washed with deionized water to remove any contamination from residual salts or amino complex. It is noted that the water bath fabrication method is highly reliable and several kinds of metal oxide nanoparticles are fabricated under different conditions.

Characterizations of the as grown copper oxide nanoparticles were performed using the following techniques. The XRD patterns were collected with a Bruker D8 advance $\mathrm{X}$-ray powder diffractometer (Bruker, Germany) equipped with a scintillation counter using $\mathrm{Cu} K \alpha$ radiation $(\lambda=$ $1.5406 \AA$ ). The goniometer scanning rate was of $0.4^{\circ} \mathrm{min}^{-1}$. The as grown nanoparticles were pressed onto a $\mathrm{Si}$ substrate before scanning. The morphology and microstructure information of as grown nanoparticles were investigated through a high-resolution transmission electron microscope (HRTEM, Tecnai G2 F20, Netherland) with a super twin objective lens and working at $200 \mathrm{kV}$ with a $\mathrm{LaB}_{6}$ filament. The influences of different temperatures and atmospheres on the electrical transport properties of annealed copper oxide nanoparticles are investigated with multiple gas mix and transport controller and a data acquisition/processing instrument (Keithley 2000 multimeter) and Agilent B1500A semiconductor device analyzer.

\section{Results and Discussion}

Figure 1 shows the XRD pattern of the as grown copper oxide nanoparticles, which indicated that the nanoparticles include $\mathrm{CuO}, \mathrm{Cu}_{2} \mathrm{O}, \mathrm{Cu}(\mathrm{OH})_{2}, \mathrm{Cu}(\mathrm{OH})_{2} \cdot \mathrm{H}_{2} \mathrm{O}$, and $\mathrm{Cu}(\mathrm{OH})_{2} \cdot 3 \mathrm{H}_{2} \mathrm{O}$ according to references PDF\#42-0638, PDF\#42-0746, PDF\#65-3288, PDF\#36-0545, and PDF\#481548, respectively. Among these nanoparticles, $\mathrm{Cu}(\mathrm{OH})_{2}$, $\mathrm{Cu}(\mathrm{OH})_{2} \cdot \mathrm{H}_{2} \mathrm{O}$, and $\mathrm{Cu}(\mathrm{OH})_{2} \cdot 3 \mathrm{H}_{2} \mathrm{O}$ are thermolabile materials, which will transform into $\mathrm{CuO}$ under relatively low heating environment. It is well known that only when the temperature is higher than $900^{\circ} \mathrm{C}$, all the $\mathrm{CuO}$ will transform into $\mathrm{Cu}_{2} \mathrm{O}$ because of the instability under high temperature.

Figure 2 shows the HRTEM analysis of as grown copper oxide nanoparticles. An irregular size of nanoparticle distributed from hundreds nanometers to micron in length and tens to hundreds nanometers in width. Under high-resolution observation, the individual nanoparticle has polycrystalline structure, the size of each crystalline grain distributed in tens nanometers scale. These nanoparticles undoubtedly have active chemical properties because of their large specific surface area, such as high surface reaction activity and efficient transmission channel for analyte molecules to reach the active sites.

Figure 3 shows the schematic diagrams of the devices based on copper oxide nanoparticles for electrical property investigation. One device was fabricated on a $\mathrm{SiO}_{2} / \mathrm{Si}$ substrate with Pt interdigital electrodes; the other was fabricated on an alumina substrate with Au interdigital electrodes. The device fabrication is as described in the following process. The copper oxide nanoparticles were initially dispersed in ethanol and then were uniformly coated onto a $300 \mathrm{~nm}$ $\mathrm{SiO}_{2} / \mathrm{Si}$ (or alumina) substrate provided with interdigitated Pt (or $\mathrm{Au}$ ) electrodes with trace and gap size of $20 \mu \mathrm{m}$ (or $250 \mu \mathrm{m}$ ) on the insulating side. In order to remove the residual water and ethanol and to ensure a good adherence of copper oxide nanoparticles to substrates and electrodes, a subsequent heating process was performed in a tube furnace. The devices were first heated at $200^{\circ} \mathrm{C}$ for 6 hours, then slowly heated up to $500^{\circ} \mathrm{C}$ for 2 hours, and finally cooled down to room temperature naturally. After the heating process, the water, crystal water, and hydroxyl inside of copper oxide nanoparticles were driven out. 


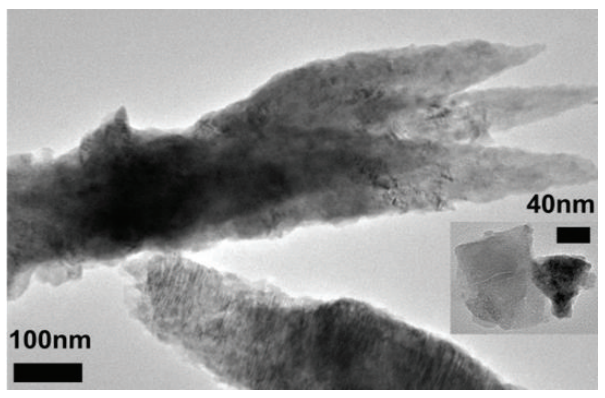

(a)

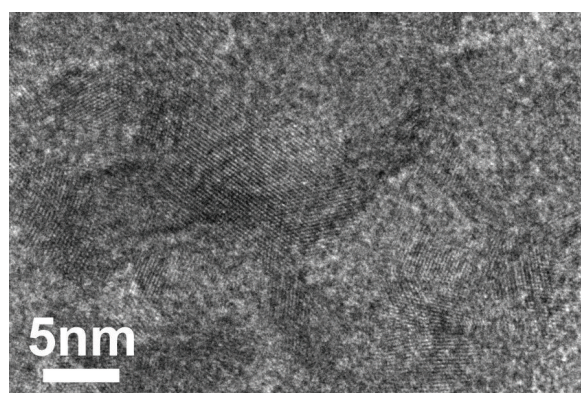

(b)

FIgURE 2: The HRTEM images of as grown copper oxide nanoparticles.
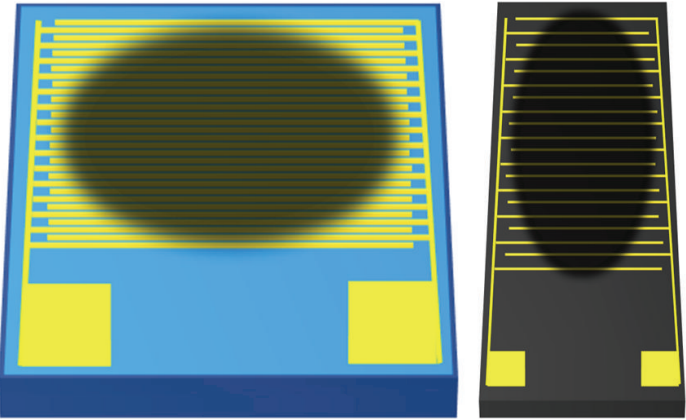

Figure 3: The schematic diagrams of the devices based on copper oxide nanoparticles.

The $I-V$ sweep measurements were performed under $\mathrm{dc}$ voltage sweep at room temperature in room air (humid), commercial air (dry), nitrogen (dry), and oxygen (dry), respectively. The voltage was scanned as $-10 \mathrm{~V} \rightarrow 0 \mathrm{~V} \rightarrow$ $10 \mathrm{~V} \rightarrow 0 \mathrm{~V} \rightarrow-10 \mathrm{~V}$ with a step of $20 \mathrm{mV}$, as shown in Figure 4(a). The $I-V$ curves in three kinds of dry gas atmosphere are similar symmetrical rectifying curves, which indicates the existence of small Schottky barriers between copper oxide nanoparticles and metal electrodes. When the voltage varied along the opposite direction, the two curves kept good coincidence with each other. However, the situation in room air atmosphere with $85 \% \mathrm{RH}$ was dramatically different. First, the current values were much larger than those measured in dry gas atmosphere. Second, when the voltage varied along the opposite direction, the two curves are entirely not coincident and show an interesting loop-like feature. The numbers $1,2,3$, and 4 indicate the voltage variation $-10 \mathrm{~V} \rightarrow 0 \mathrm{~V}, 0 \mathrm{~V} \rightarrow 10 \mathrm{~V}, 10 \mathrm{~V} \rightarrow 0 \mathrm{~V}$, and $0 \mathrm{~V} \rightarrow-10 \mathrm{~V}$, respectively. The curve segments 1 and 3 indicate that when the voltage got smaller, the current also got smaller in the meantime, which is apparently reasonable. Yet the curve segments 2 and 4 are very different; in detail, the current got smaller when voltage got larger than $-5 \mathrm{~V}$ and $5 \mathrm{~V}$.

The only difference between the humid room air atmosphere and commercial dry air atmosphere is in the water vapor. The contrast experiments show the dramatic influence of water vapor on the electrical conductance of copper oxide nanoparticles. It is well known that in a water molecule, the oxygen atom and two hydrogen atoms share electrons in covalent bonds, but the sharing is not equal. In the covalent bond between oxygen and hydrogen, the oxygen atom attracts electrons stronger than the hydrogen atoms. The unequal sharing of electrons gives the water molecule a slight negative charge near its oxygen atom and a slight positive charge near its hydrogen atoms [15]. As a result, the polar water molecules in humid air and the electrons on the surface of copper oxide nanoparticles will adsorb each other. More and more water molecules were attracted to form an ultrathin water layer in the surfaces of copper oxide nanoparticles. In this water layer, the following hydrolysis reaction and ionization process might happen:

$$
\begin{aligned}
& \mathrm{CuO}+\mathrm{H}_{2} \mathrm{O} \longrightarrow \mathrm{Cu}(\mathrm{OH})_{2} \\
& \mathrm{Cu}(\mathrm{OH})_{2} \longrightarrow \mathrm{Cu}^{2+}+2 \mathrm{OH}^{-}
\end{aligned}
$$

Both positive and negative ions will enhance the conductance. When the speed of water molecules aggregation is faster than speed of hydrolysis reaction and ionization process, the current may become saturated and even smaller because the concentration variation of ions may reach a bottleneck, as Figure 4(a) shows.

As shown in Figure 4(b), the dc sweep voltage was scanned as $-10 \mathrm{~V} \rightarrow 0 \mathrm{~V} \rightarrow 10 \mathrm{~V} \rightarrow 0 \mathrm{~V} \rightarrow-10 \mathrm{~V}$ with different voltage variation step value of $50 \mathrm{mV}, 100 \mathrm{mV}$, $150 \mathrm{mV}, 200 \mathrm{mV}$, and $250 \mathrm{mV}$ in room air atmosphere with $85 \% \mathrm{RH}$, respectively. With increasement of voltage variation step value, the loop-like curve became thinner and thinner. Because the voltage variation step value decided the entire sweeping time, the larger variation step value resulted in the shorter entire sweeping time and the less adsorbed water molecules; the smaller variation step value resulted in the longer entire sweep time and the more adsorbed water molecules. This theory can well explain the different shapes of $I-V$ curves.

In order to investigate the influence of oxygen adsorption on the electrical conductivity of copper oxide nanoparticles at high temperature, a gas sensor on alumina substrate provided with interdigitated Au electrodes (trace and gap size: $250 \mu \mathrm{m}$ ) on its surface through aforementioned process is fabricated. Before the coating process, fine gold wires $(100 \mu \mathrm{m}$ in diameter) were connected to the interdigitated 


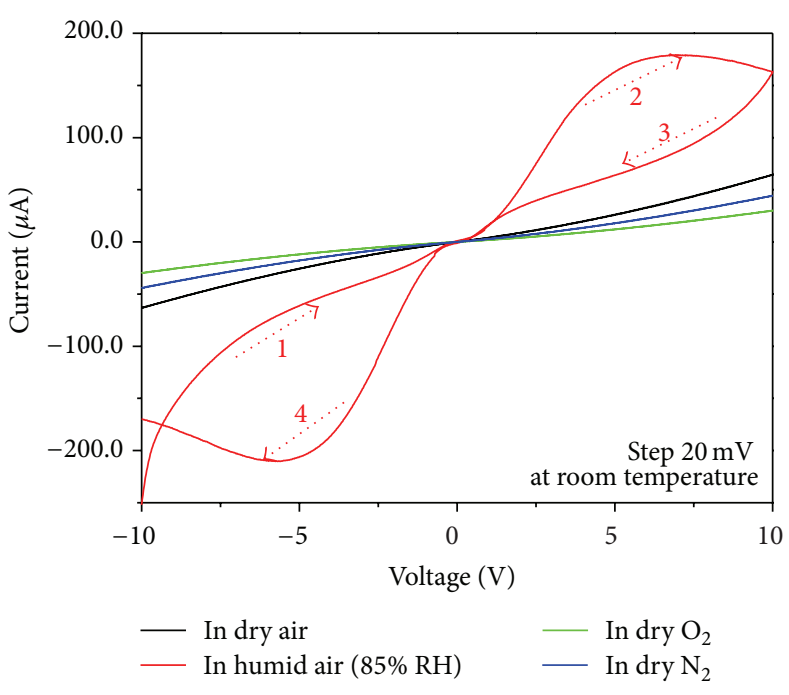

(a)

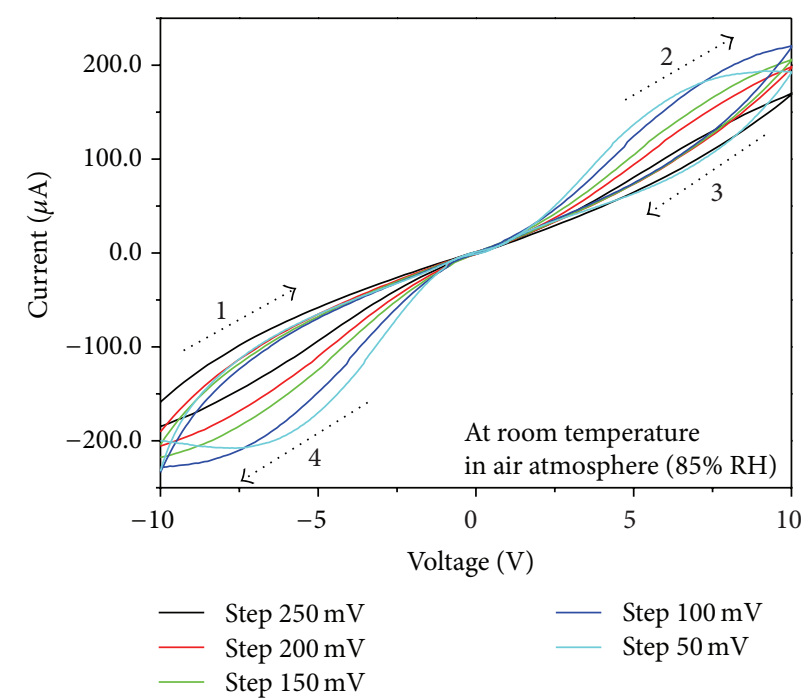

(b)

FIgURE 4: (a) Current variation of copper oxide nanoparticles under voltage from $-10 \mathrm{~V}$ to $10 \mathrm{~V}$ to $-10 \mathrm{~V}$ under different atmospheres; (b) current variation of copper oxide nanoparticles under voltage from $-10 \mathrm{~V}$ to $10 \mathrm{~V}$ to $-10 \mathrm{~V}$ with different voltage variation step values in humid air atmosphere.

$\mathrm{Au}$ electrodes in assistance of gold glue, with a subsequent thermal solidification treatment $\left(800^{\circ} \mathrm{C}\right.$ for $\left.60 \mathrm{~min}\right)$.

Figure 5(a) shows the $I-V$ character of the copper oxide nanoparticles from $-40 \mathrm{~V}$ to $40 \mathrm{~V}$ with a step of $50 \mathrm{mV}$ in commercial dry air atmosphere at different temperatures of $100^{\circ} \mathrm{C}, 150^{\circ} \mathrm{C}, 200^{\circ} \mathrm{C}$, and $250^{\circ} \mathrm{C}$, respectively. The figure shows that the resistance of the copper oxide nanoparticles is highly sensitive to temperature. Figure 5(b) shows the $I$ $V$ character of the copper oxide nanoparticles from $-40 \mathrm{~V}$ to $40 \mathrm{~V}$ with a step of $50 \mathrm{mV}$ at $200^{\circ} \mathrm{C}$ in different mixture gas atmospheres with different oxygen concentrations of $5 \%, 10 \%, 15 \%$, and $20 \%$, respectively. At the same voltage, the current increased along with the increase of oxygen concentration. Figure 5(c) shows the time-resolved current (or resistance) variation of copper oxide nanoparticles in different mixture gas atmospheres with oxygen concentration of $5 \%, 10 \%, 15 \%$ s and $20 \%$, respectively. The voltage is $10 \mathrm{~V}$ and the temperature is $200^{\circ} \mathrm{C}$. One can observe that each current curve slowly increases along with the time due to an increasing oxygen adsorption. It is also obvious that the current level of four curves got a monotonous increase along with the increasing density of oxygen, which is also due to the oxygen adsorption. Because copper oxide is well known as a p-type semiconductor, the majority carrier is hole and the minority carrier is electron. The adsorption of oxygen will decrease the electron concentration and increase the hole concentration in copper oxide, which makes the resistance decrease.

The HCHO sensing properties were investigated at atmospheric pressure through a dynamic test system which included a horizontal tube furnace, a multigas mix and transport controller and a data acquisition/processing instrument
(Keithley 2000 multimeter), using a constant synthetic air flow $(100 \mathrm{sccm})$ as both reference gas and carrier gas for the analyte dispersion. Time dependencies of the resistance variation at different temperatures $\left(300^{\circ} \mathrm{C}\right.$ and $\left.400^{\circ} \mathrm{C}\right)$ of copper oxide nanoparticles upon exposure towards 4 pulses of $\mathrm{HCHO}(10,20,30$, and $40 \mathrm{ppm})$ in dry air are shown in Figure 6 . The results presented are highly reproducible on similar devices. The exposure time in $\mathrm{HCHO}$ is 4 minutes and the intermission time in air is 6 minutes. The applied voltage between the double Au electrodes is kept at $15 \mathrm{~V}$ during the entire process.

At both temperatures the resistance of copper oxide nanoparticles gradually increased during sensing of $\mathrm{HCHO}$ and gradually decreased since $\mathrm{HCHO}$ stopped. The copper oxide nanoparticles showed stable p-type conductivity at both $300^{\circ} \mathrm{C}$ and $400^{\circ} \mathrm{C}$ according to the decrease of resistance when the environment atmosphere is air. The sensing mechanism of resistive gas sensors based on metal oxide semiconductors is ascribed to variations of majority carrier surface concentration under the influence of adsorbed substances [16]. Particularly, the surface depletion model and grain boundary model are used to describe the sensing mechanism of metal oxide semiconductors [17]. For ptype metal oxide semiconductors, the adsorbed oxygen will form acceptor levels near the valence band at the surface, resulting in the upward band bending and formation of an accumulation layer of holes. The upward band bending between nanoparticles will enhance the transport of holes, the majority carriers in p-type metal oxide semiconductors. During exposing to reductive gas, the reductive gas molecules would react with the preadsorbed oxygen species, resulting in the cancellation of holes and an increase of the resistance. 


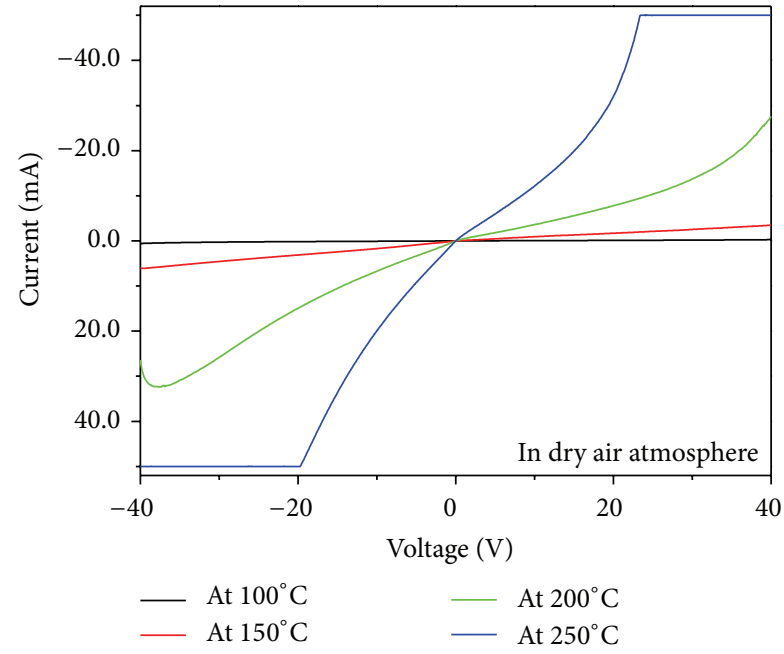

(a)

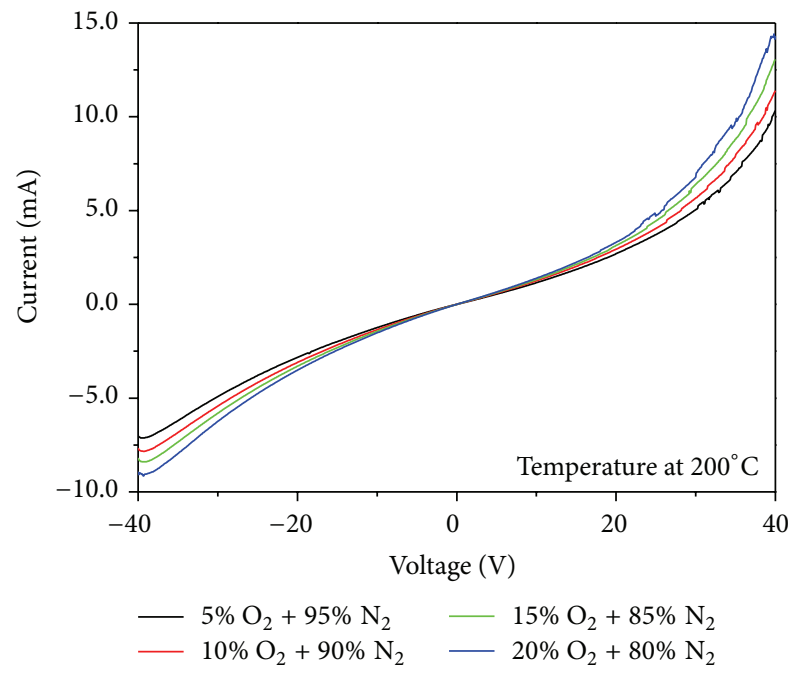

(b)

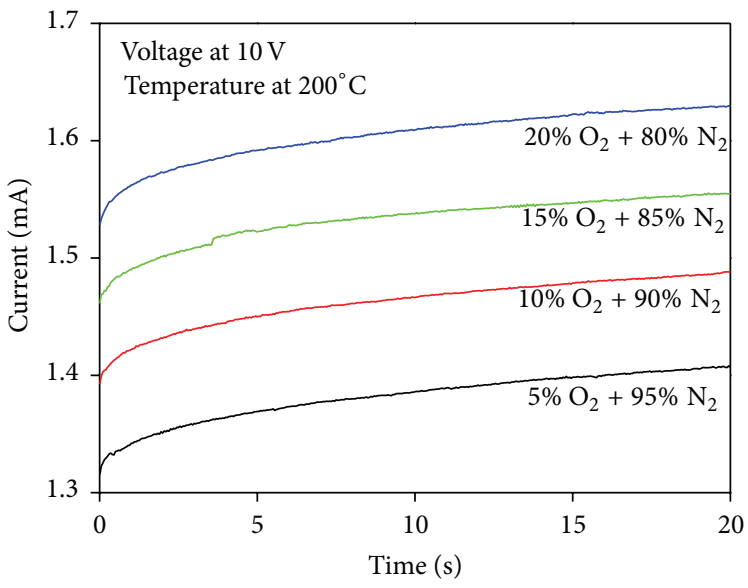

(c)

FiguRE 5: (a) $I-V$ character of the copper oxide nanoparticles under voltage from $-40 \mathrm{~V}$ to $40 \mathrm{~V}$ with a step of $50 \mathrm{mV}$ in dry air atmosphere at different temperatures; (b) $I-V$ character of the copper oxide nanoparticles at $200^{\circ} \mathrm{C}$ in different atmospheres; (c) time-resolved current variation of copper oxide nanoparticles in different atmospheres.

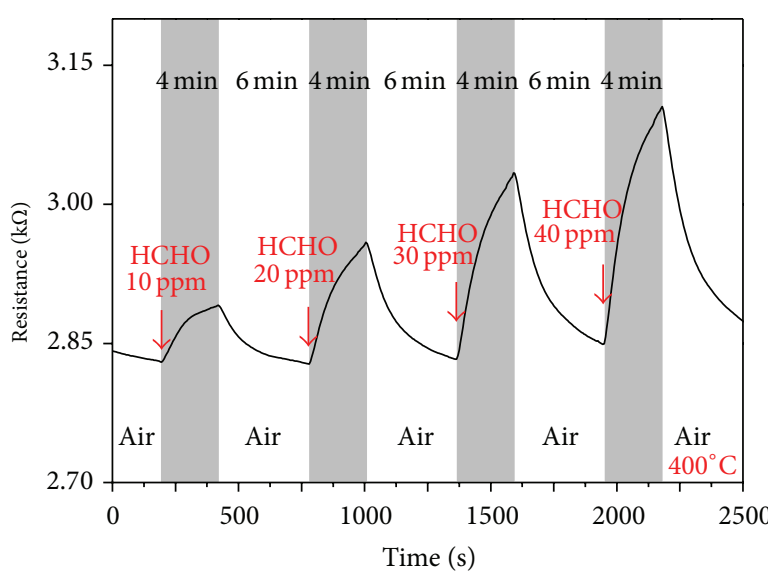

(a)

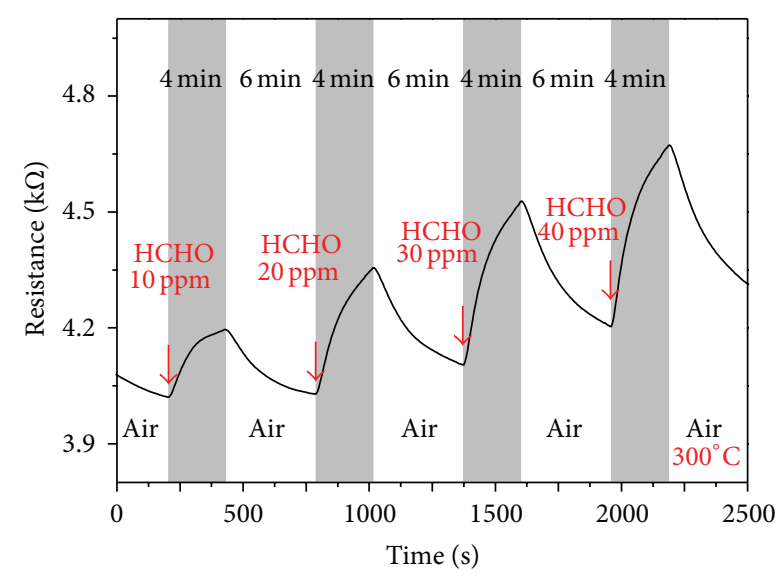

(b)

FIGURE 6: The HCHO sensitivity of copper oxide nanoparticles at different temperatures. 
This theory can very well explain the variation of resistance of copper oxide nanoparticles during sensing $\mathrm{HCHO}$ at $300^{\circ} \mathrm{C}$ and $400^{\circ} \mathrm{C}$.

\section{Conclusions}

In conclusion, we have synthesized copper oxide nanoparticles and investigated the influence of different atmospheres and temperatures on their electrical conductivity. We found that the $I-V$ character of copper oxide nanoparticles is very sensitive to humid air atmosphere with $85 \% \mathrm{RH}$ at room temperature and also sensitive to the variation of temperature and oxygen concentration. Further, at high temperature they are very sensitive to $\mathrm{HCHO}$ concentration variation.

\section{Conflict of Interests}

The authors declare that there is no conflict of interests regarding the publication of this paper.

\section{Acknowledgments}

This work was supported by the National Natural Science Foundation of China (Grant nos. 61107028, 61131004, 61001054, and 61274076) and the Fundamental Research Funds for the Central Universities (Grant no. DUT12JB11).

\section{References}

[1] J. Xu, Q. Pan, Y. Shun, and Z. Tian, "Grain size control and gas sensing properties of $\mathrm{ZnO}$ gas sensor," Sensors and Actuators B, vol. 66, no. 1, pp. 277-279, 2000.

[2] F. Zhang, H. Yang, X. Xie et al., "Controlled synthesis and gassensing properties of hollow sea urchin-like $\alpha$ - $\mathrm{Fe}_{2} \mathrm{O}_{3}$ nanostructures and $\alpha-\mathrm{Fe}_{2} \mathrm{O}_{3}$ nanocubes," Sensors and Actuators B, vol. 141, no. 2, pp. 381-389, 2009.

[3] Y.-J. Choi, I.-S. Hwang, J.-G. Park, K. J. Choi, J.-H. Park, and J.-H. Lee, "Novel fabrication of an $\mathrm{SnO}_{2}$ nanowire gas sensor with high sensitivity," Nanotechnology, vol. 19, no. 9, Article ID 095508, 2008.

[4] L. You, Y. Sun, J. Ma et al., "Highly sensitive $\mathrm{NO}_{2}$ sensor based on square-like tungsten oxide prepared with hydrothermal treatment," Sensors and Actuators B, vol. 157, no. 2, pp. 401-407, 2011.

[5] X. Chu, S. Liang, W. Sun, W. Zhang, T. Chen, and Q. Zhang, "Trimethylamine sensing properties of sensors based on $\mathrm{MoO}_{3}$ microrods," Sensors and Actuators B, vol. 148, no. 2, pp. 399-403, 2010.

[6] Y. Zhang, X. He, J. Li, H. Zhang, and X. Gao, "Gas-sensing properties of hollow and hierarchical copper oxide microspheres," Sensors and Actuators B, vol. 128, no. 1, pp. 293-298, 2007.

[7] H. Steinebach, S. Kannan, L. Rieth, and F. Solzbacher, " $\mathrm{H}_{2}$ gas sensor performance of $\mathrm{NiO}$ at high temperatures in gas mixtures," Sensors and Actuators B, vol. 151, no. 1, pp. 162-168, 2010.

[8] R. K. Bedi and I. Singh, "Room-temperature ammonia sensor based on cationic surfactant-assisted nanocrystalline $\mathrm{CuO}$," ACS Applied Materials and Interfaces, vol. 2, no. 5, pp. 1361-1368, 2010.
[9] B. J. Hansen, N. Kouklin, G. Lu, I.-K. Lin, J. Chen, and X. Zhang, "Transport, analyte detection, and opto-electronic response of p-type CuO nanowires," Journal of Physical Chemistry C, vol. 114, no. 6, pp. 2440-2447, 2010.

[10] C. Yang, X. Su, F. Xiao, J. Jian, and J. Wang, "Gas sensing properties of $\mathrm{CuO}$ nanorods synthesized by a microwaveassisted hydrothermal method," Sensors and Actuators B, vol. 158, no. 1, pp. 299-303, 2011.

[11] X. Gou, G. Wang, J. Yang, J. Park, and D. Wexler, "Chemical synthesis, characterisation and gas sensing performance of copper oxide nanoribbons," Journal of Materials Chemistry, vol. 18, no. 9, pp. 965-969, 2008.

[12] F. Zhang, A. Zhu, Y. Luo, Y. Tian, J. Yang, and Y. Qin, “CuO nanosheets for sensitive and selective determination of $\mathrm{H}_{2} \mathrm{~S}$ with high recovery ability," Journal of Physical Chemistry C, vol. 114, no. 45, pp. 19214-19219, 2010.

[13] Y. Zhang, X. He, J. Li, H. Zhang, and X. Gao, "Gas-sensing properties of hollow and hierarchical copper oxide microspheres," Sensors and Actuators B, vol. 128, no. 1, pp. 293-298, 2007.

[14] K. Keis, L. Vayssieres, S.-E. Lindquist, and A. Hagfeldt, "Nanostructured $\mathrm{ZnO}$ electrodes for photovoltaic applications," Nanostructured Materials, vol. 12, no. 1, pp. 487-490, 1999.

[15] The American Chemical Society, Middle School Chemistry, chapter 5, Water is a Polar Molecule, http://www.middleschoolchemistry.com/lessonplans/chapter5/lesson1.

[16] W. Göpel and K. D. Schierbaum, "Electronic conductance and capacitance sensors," in A Comprehensive Survey, W. Göpel, J. Hesse, and J. N. Zemel, Eds., vol. 2, VCH Weinheim, New York, NY, USA, 1991.

[17] F. P. Koffyberg and F. A. Benko, "A photoelectrochemical determination of the position of the conduction and valence band edges of $p$-type CuO," Journal of Applied Physics, vol. 53, no. 2, pp. 1173-1177, 1982. 

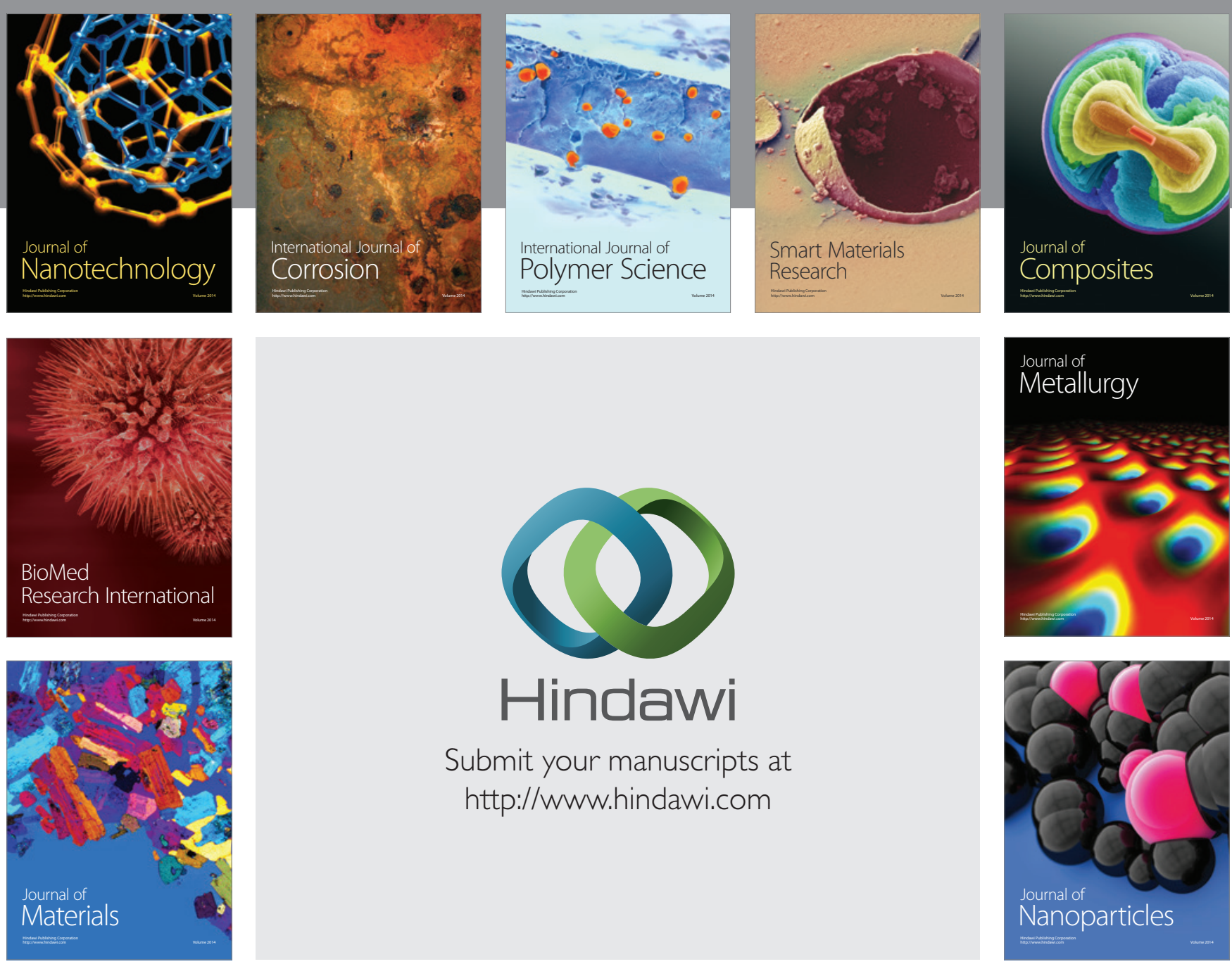

Submit your manuscripts at http://www.hindawi.com
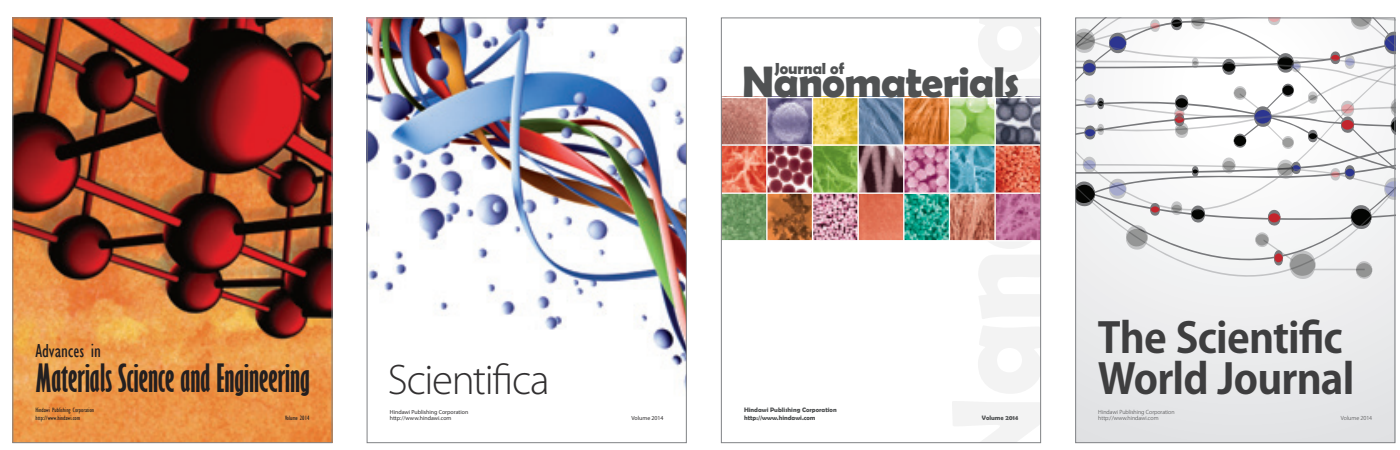

\section{The Scientific World Journal}
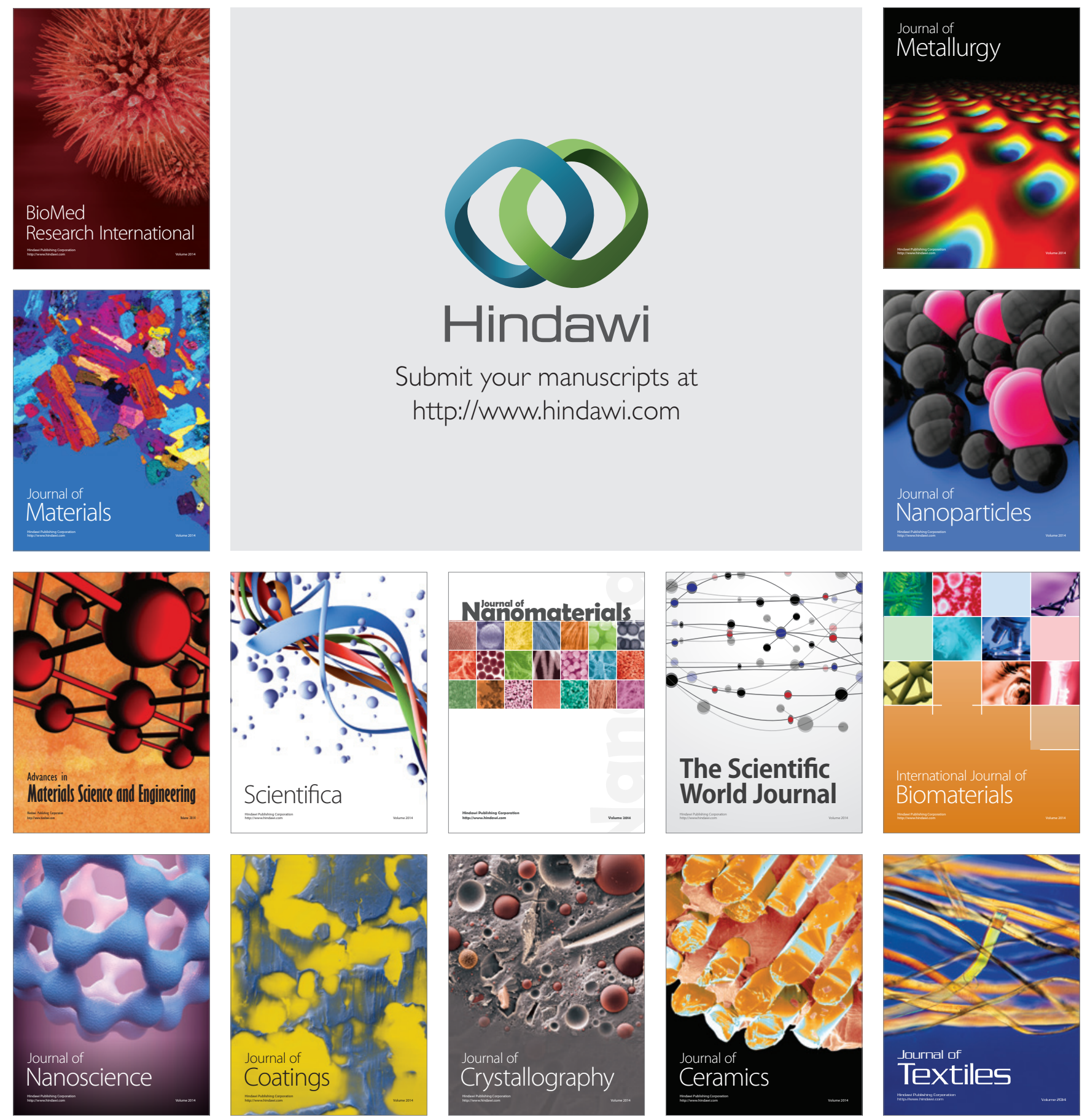